\title{
高暴露(001)面 $\mathrm{BiOBr} / \mathrm{Ti}_{3} \mathrm{C}_{2}$ 复合光催化剂的制备 及其可见光催化性能
}

\author{
李志锋 ${ }^{1}$, 谭 杰 $^{1}$, 杨晓飞 ${ }^{1}$, 萄祖弘 ${ }^{1}$, 狍正来 ${ }^{1}$, 张婷婷 ${ }^{1,2}$ \\ (1. 北京化工大学 化学工程学院, 北京 $100029 ; 2$. 北京市环境污染控制与资源化工程研究中心, 北京 100029)
}

摘 要: 通过水解法合成具有高暴露(001)面的 $\mathrm{BiOBr} / \mathrm{Ti}_{3} \mathrm{C}_{2}$ 复合光催化剂, 利用不同手段对样品进行表征。以罗丹 明 $\mathrm{B}$ 为目标污染物, 评价了不同样品的可见光催化性能。结果表明, 层状 $\mathrm{Ti}_{3} \mathrm{C}_{2}$ 添加量为 $20.0 \mathrm{wt} \%$ 时, $\mathrm{BiOBr} / \mathrm{Ti}_{3} \mathrm{C}_{2}$ 复合光催化剂在 $60 \mathrm{~min}$ 内对罗丹明 $\mathrm{B}$ 的降解效率为 $97.1 \%$, 比 $\mathrm{BiOBr}$ 的降解效率提高了 $34.7 \%$ 。引入层状 $\mathrm{Ti}_{3} \mathrm{C}_{2}$ 使得 $\mathrm{BiOBr}$ 与 $\mathrm{Ti}_{3} \mathrm{C}_{2}$ 界面形成肖特基结能垒, 产生有效的电子陷阱抑制了光生电子-空穴对的复合, 从而大大提高 了 $\mathrm{BiOBr}$ 的可见光催化活性。 $\mathrm{BiOBr} / \mathrm{Ti}_{3} \mathrm{C}_{2}$ 复合光催化剂经 5 次循环实验后，降解效率仍保持在 $91.0 \%$, 表明其具 有良好的稳定性。活性物种捕获实验表明，超氧自由基为罗丹明 $\mathrm{B}$ 可见光催化降解中的主要活性物种，并据此提出 了可能的光催化机理。

关 键 词: $\mathrm{BiOBr} ; \mathrm{Ti}_{3} \mathrm{C}_{2}$; 可见光催化; 肖特基结

中图分类号: TQ174 文献标识码: A

\section{Preparation and Visible Light Photocatalytic Performance of $\mathrm{BiOBr} / \mathrm{Ti}_{3} \mathrm{C}_{2}$ Composite Photocatalyst with Highly Exposed (001) Facets}

\author{
LI Zhifeng ${ }^{1}$, TAN Jie ${ }^{1}$, YANG Xiaofei ${ }^{1}$, LIN Zuhong ${ }^{1}$, HUAN Zhenglai ${ }^{1}$, ZHANG Tingting ${ }^{1,2}$
}

(1. College of Chemical Engineering, Beijing University of Chemical Technology, Beijing 100029, China; 2. Beijing Centre for Resource and Environmental Research, Beijing 100029, China)

\begin{abstract}
BiOBr} / \mathrm{Ti}_{3} \mathrm{C}_{2}$ composite photocatalyst with highly exposed (001) facets was synthesized by hydrolysis method. Different instruments were employed to characterize the samples. The visible light photocatalytic performance of different samples were evaluated by using Rhodamine B as the target pollutant. The results show that the degradation efficiency of Rhodamine $\mathrm{B}$ reaches $97.1 \%$ within $60 \mathrm{~min}$ over $\mathrm{BiOBr} / \mathrm{Ti}_{3} \mathrm{C}_{2}\left(20.0 \mathrm{wt} \% \mathrm{Ti}_{3} \mathrm{C}_{2}\right.$ addidion) composite photocatalyst, which is $34.7 \%$ higher than that of $\mathrm{BiOBr}$. With the introduction of layered $\mathrm{Ti}_{3} \mathrm{C}_{2}$, the interface between $\mathrm{BiOBr}$ and $\mathrm{Ti}_{3} \mathrm{C}_{2}$ forms the Schottky junction energy barrier, which produces effective electron traps to inhibit the combination of photogenic electron-hole pairs, and greatly improves the visible light photocatalytic activity of $\mathrm{BiOBr}$. After 5 cycles, the degradation efficiency of $\mathrm{BiOBr} / \mathrm{Ti}_{3} \mathrm{C}_{2}$ composite photocatalyst remains $91.0 \%$, showing reliable stability. The active species capture experiment shows that superoxide radical $\left(\cdot \mathrm{O}_{2}^{-}\right)$is the main active species in the photocatalytic degradation of Rhodamine $\mathrm{B}$, and a possible photocatalytic mechanism is proposed accordingly.
\end{abstract}

收稿日期: 2019-12-11; 收到修改稿日期：2020-02-10

基金项目：国家自然科学基金(41977142); 水体污染控制与治理科技重大专项(2018ZX07111003)

National Natural Science Foundation of China (41977142); Major Science and Technology Project for Water Pollution Control and Treatment (2018ZX07111003)

作者简介: 李志锋(1996-), 男, 硕士研究生. E-mail: lzf260cas@163.com LI Zhifeng(1996-), male, Master candidate. E-mail: lzf260cas@163.com

通讯作者: 张婷婷, 副教授. E-mail: zhangtt@mail.buct.edu.cn ZHANG Tingting, associate professor. E-mail: zhangtt@mail.buct.edu.cn 
Key words: $\mathrm{BiOBr} ; \mathrm{Ti}_{3} \mathrm{C}_{2}$; visible light photocatalysis; Schottky junction

$\mathrm{TiO}_{2}$ 具有物理和化学性质稳定、光催化活性高、 无毒及成本低等优点, 得到了广泛研究 ${ }^{[1-2]}$ 。但是, $\mathrm{TiO}_{2}$ 禁带宽度为 $3.2 \mathrm{eV}$, 属于宽带隙半导体, 只能 吸收占太阳光谱 $5 \%$ 的紫外光, 极大地限制了其实 际应用 ${ }^{[3]}$ 。因此, 开发具有优异可见光催化活性的光 催化剂逐渐成为研究热点。

近年来, $\mathrm{BiOBr}$ 可见光催化剂逐渐引起关注, 它 具有正方氟氯铅矿结构, 属于四方晶系 ${ }^{[4]}$ 。铋氧层 $\left(\left[\mathrm{Bi}_{2} \mathrm{O}_{2}\right]^{2+}\right)$ 和卤素层 $\left(\mathrm{X}^{-}\right)$的交替排列, 具有开放式的 晶体结构和间接跃迁的带隙结构, 这种独特的结构 性质使光生电子-空穴对可以得到有效分离, 从而 提高 $\mathrm{BiOBr}$ 的光催化活性 ${ }^{[5-6]}$ 。 $\mathrm{BiOBr}$ 具有合适的带 隙宽度和可见光响应能力 ${ }^{[7]}$, 但是对可见光利用率 有限, 光生载流子的分离效率还有待提高 ${ }^{[8]}$ 。为此, 研究者分别从形貌调控、晶面调控、引入助催化剂和 构建异质结体系等方面进行了大量的改性研究 ${ }^{[8-11]}$, 以提高 $\mathrm{BiOBr}$ 的可见光催化活性。

$\mathrm{Ti}_{3} \mathrm{C}_{2}$ 是一种新型二维过渡金属碳化物，具有良 好的导电性、优异的化学结构稳定性和亲水性, 得 到了催化领域研究者的广泛关注, 被大量应用于与 其他半导体光催化剂复合构建异质结来提高催化活 性。密度泛函理论计算表明 $\mathrm{Ti}_{3} \mathrm{C}_{2}$ 具有突出的金属 导电性 ${ }^{[12]}$, 在与其他半导体界面的连接处易形成肖 特基势垒, 内建电场的产生有效地改善了光催化剂 光生电子-空穴对的分离, 从而提高其催化活性 ${ }^{[13]}$ 。 Peng 等 ${ }^{[14]}$ 以 $\mathrm{Ti}_{3} \mathrm{C}_{2}$ 作为钛源, 通过水热法原位生长 具有高暴露(001)面的 $\mathrm{TiO}_{2}$, 界面肖特基结的形成改 善了 $\mathrm{TiO}_{2}$ 对甲基橙染料的降解效果。Cai 等 ${ }^{[15]}$ 通过 静电自组装方法合成了 $\mathrm{Ag}_{3} \mathrm{PO}_{4} / \mathrm{Ti}_{3} \mathrm{C}_{2}$ 肖特基结光催 化剂, 与 $\mathrm{Ag}_{3} \mathrm{PO}_{4} / \mathrm{RGO}$ 和 $\mathrm{Ag}_{3} \mathrm{PO}_{4}$ 光催化剂相比, $\mathrm{Ag}_{3} \mathrm{PO}_{4} / \mathrm{Ti}_{3} \mathrm{C}_{2}$ 光催化剂降解 2,4-二硝基苯酚的表观 速率分别提高了 1.5 和 9 倍。近期, 也有少量研究表 明, 通过 $\mathrm{BiOBr}$ 和 $\mathrm{Ti}_{3} \mathrm{C}_{2}$ 复合催化剂的构建, 可以提 升 $\mathrm{BiOBr}$ 对有机污染物的光催化降解速率及产氢效 率 ${ }^{[16-18]}$ 。由此可见, $\mathrm{Ti}_{3} \mathrm{C}_{2}$ 作为助催化剂具有较好的 应用前景, 但还未见高暴露(001)面 $\mathrm{BiOBr}$ 与 $\mathrm{Ti}_{3} \mathrm{C}_{2}$ 复合催化剂的改性研究报道。

本研究通过简易水解法合成具有高暴露 (001) 面的 $\mathrm{BiOBr} / \mathrm{Ti}_{3} \mathrm{C}_{2}$ 复合光催化剂, 通过引入不同量 $\mathrm{Ti}_{3} \mathrm{C}_{2}$ 确定最佳复合比例, 采用多种表征手段对催化 剂结构和光学特性进行分析, 最后通过对罗丹明 $\mathrm{B}$ 染料废水的降解和循环实验来评价催化剂的性能和 稳定性。

\section{1 实验方法}

\section{1 原料与试剂}

五水硝酸铋、澳化钾、罗丹明 B、氢氟酸、对 苯醌、草酸铵均购自阿拉丁生化科技股份有限公司, 异丙醇购自麦克林生化科技股份有限公司, $\mathrm{Ti}_{3} \mathrm{AlC}_{2}$ 购自吉林 11 科技有限公司。药品均为分析纯, 使用 过程中不需要进一步提纯。实验用水为去离子水。

\section{2 材料制备}

\subsection{1 $\mathrm{Ti}_{3} \mathrm{C}_{2}$ 的制备}

通过 $\mathrm{HF}$ 刻蚀 $\mathrm{Ti}_{3} \mathrm{AlC}_{2}$ 制备层状 $\mathrm{Ti}_{3} \mathrm{C}_{2}$ 纳米片: 将 $1.0 \mathrm{~g} \mathrm{Ti}_{3} \mathrm{AlC}_{2}$ 粉末置于氢氟酸 $(30 \mathrm{~mL}, 49 \mathrm{wt} \%)$ 溶液 中, 二者混合后在 $35{ }^{\circ} \mathrm{C}$ 下连续搅拌 $24 \mathrm{~h}$, 然后用去 离子水反复洗涤混合溶液至中性, 并离心收集层状 $\mathrm{Ti}_{3} \mathrm{C}_{2}$ 粉末, 最后在 $60{ }^{\circ} \mathrm{C}$ 下真空干燥 $12 \mathrm{~h}$ 备用。

\subsection{2 高暴露和非高暴露(001)面 $\mathrm{BiOBr}$ 的制备}

首先将 $1.0 \mathrm{mmol} \mathrm{KBr}$ 溶解于 $15 \mathrm{~mL}$ 去离子水中, 得到 $\mathrm{A}$ 溶液, 然后将 $1.0 \mathrm{mmol} \mathrm{Bi}\left(\mathrm{NO}_{3}\right)_{3} \cdot 5 \mathrm{H}_{2} \mathrm{O}$ 分散 于 $15 \mathrm{~mL}$ 无水乙醇中, 超声分散得到 $\mathrm{B}$ 悬浮液。 $\mathrm{A}$ 溶液加热至 $90{ }^{\circ} \mathrm{C}$ 时, 将上述 $\mathrm{B}$ 溶液滴入 $\mathrm{A}$ 溶液中, 搅拌均匀后在 $90{ }^{\circ} \mathrm{C}$ 反应 $3 \mathrm{~h}$ 。反应结束后冷却至室 温, 过滤收集 $\mathrm{BiOBr}$, 然后在 $70{ }^{\circ} \mathrm{C}$ 下真空干燥 $10 \mathrm{~h}$, 制得高暴露(001)面 $\mathrm{BiOBr}$ ，记为 $\mathrm{BiOBr}$ 。

将 $15 \mathrm{~mL}$ 无水乙醇换为 $15 \mathrm{~mL}$ 去离子水, 按同 样步骤制得非高暴露(001)面 $\mathrm{BiOBr}$, 记为 $\mathrm{FBiOBr}$ 。

\subsection{3 高暴露和非高暴露 $(001)$ 面 $\mathrm{BiOBr} / \mathrm{Ti}_{3} \mathrm{C}_{2}$ 的} 制备

首先将一定量层状 $\mathrm{Ti}_{3} \mathrm{C}_{2}$ 分散于上述 $\mathrm{A}$ 溶液中, 加热至 $90{ }^{\circ} \mathrm{C}$ 时, 将 $\mathrm{B}$ 溶液滴入 $\mathrm{A}$ 溶液, 在 $90{ }^{\circ} \mathrm{C}$ 下 反应 $3 \mathrm{~h}$ 。反应结束后冷却至室温, 过滤收集 $\mathrm{BiOBr} / \mathrm{Ti}_{3} \mathrm{C}_{2}$ 复合物, 然后在 $70{ }^{\circ} \mathrm{C}$ 下真空干燥 $10 \mathrm{~h}$ 。 制得的高暴露(001)面 $\mathrm{BiOBr} / \mathrm{Ti}_{3} \mathrm{C}_{2}$ 复合光催化剂, 按层状 $\mathrm{Ti}_{3} \mathrm{C}_{2}$ 添加量的不同 $(10.0 \mathrm{wt} \% 、 20.0 \mathrm{wt} \%$ 、 $30.0 \mathrm{wt} \%)$ 分别记为 BT-10、BT-20、BT-30。

按照上述步骤制备非高暴露 (001) 面 $\mathrm{BiOBr} / \mathrm{Ti}_{3} \mathrm{C}_{2}$ 复合光催化剂, 层状 $\mathrm{Ti}_{3} \mathrm{C}_{2}$ 添加量为 $20.0 \mathrm{wt} \%$ 的记为 FBT- 20 。

\section{3 材料表征}

采用 X 射线衍射仪(D8 Advance, 德国 BrukerAXS 公司)对粉末材料的晶体物相进行表征, 靶源 为 $\mathrm{Cu} \mathrm{Ka}$ 射线, 波长 $\lambda$ 为 $0.15406 \mathrm{~nm}$, 测试电压为 $40.0 \mathrm{kV}$ 、电流为 $40.0 \mathrm{~mA}$, 扫描范围为 $5^{\circ} \sim 90^{\circ}$ 。通 
过 X 射线光电子能谱仪(ESCALAB 250XI, 美国赛 默飞公司)测定样品的化学组成, 以 $\mathrm{C} 1 \mathrm{~s}$ 位于 $284.8 \mathrm{eV}$ 处的表面污染碳作为结合能的校正基准。采用扫描 电子显微镜(Gemini SEM 300/VP, 德国卡尔蔡司公 司)、透射电子显微镜(JEM-2010, 日本电子)和能谱 仪(EDS)表征样品的微观形貌和元素组成。利用紫 外-可见漫反射光谱仪(UV-3600, 日本岛津公司)获 取样品光学性质, 以 $\mathrm{BaSO}_{4}$ 作为参比。利用荧光光 度计(F-7000, 日本日立公司)测试样品的发光性能, 激发波长为 $350 \mathrm{~nm}$ 。

\section{4 光催化性能实验}

通过在可见光照射条件下降解罗丹明 $\mathrm{B}(\mathrm{RhB})$ 溶液来测定光催化剂的光催化活性。将 $50.0 \mathrm{mg}$ 的 样品分散于 $100 \mathrm{~mL} 20 \mathrm{mg} / \mathrm{L}$ 的 $\mathrm{RhB}$ 溶液中, 在避光 条件下搅拌 $60 \mathrm{~min}$ 以达到吸附一解吸平衡。可见光 源为 $500 \mathrm{~W}$ 的氙灯, 并用滤光片滤去波长小于 $420 \mathrm{~nm}$ 的光。在光照条件下, 每间隔 $15 \mathrm{~min}$ 取 $5 \mathrm{~mL}$ 上清液, 在 $\mathrm{RhB}$ 的最大吸收波长 $554 \mathrm{~nm}$ 处测定吸 光度, 然后通过朗伯一比尔公式计算 $\mathrm{RhB}$ 的降解率:

$$
\eta=\left(C_{0}-C_{t}\right) / C_{0} \times 100 \%
$$

其中: $\eta$ 为降解率; $C_{0}$ 为暗吸附平衡后 $\mathrm{RhB}$ 溶液的吸 光度; $C_{t}$ 为某时刻光照反应后 $\mathrm{RhB}$ 溶液的吸光度。

\section{2 结果与讨论}

\section{1 表征结果}

\subsubsection{XRD 分析}

通过 XRD 分析样品的晶体结构, 从图 1 中可 见, MAX 相 $\mathrm{Ti}_{3} \mathrm{AlC}_{2}$ 在 $2 \theta=39.05^{\circ}$ 处出现的较强衍射 峰 ${ }^{[19]}$, 对应 $\mathrm{Ti}_{3} \mathrm{AlC}_{2}$ 的(104)晶面, 经过 $\mathrm{HF}$ 刻蚀之后, (104) 晶面衍射峰完全消失, 位于 $2 \theta=9.32^{\circ}$ 处的 $(002)$ 晶面衍射峰和 $2 \theta=19.07^{\circ}$ 的(004)晶面衍射峰加宽并 向低角度移动, 表明刻蚀后的 $\mathrm{Ti}_{3} \mathrm{AlC}_{2}$ 成功去除了 夹层 $\mathrm{Al}$, 并且完成了向层状 $\mathrm{Ti}_{3} \mathrm{C}_{2}$ 的转变 ${ }^{[20]}$ 。由 图 1(a)可知, $\mathrm{BiOBr}$ 和 $\mathrm{FBiOBr}$ 具有窄而尖的衍射峰, 表明样品的结晶性较好, 且所有衍射峰与四方相 $\mathrm{BiOBr}$ 的衍射峰(JCPDS 09-0393)一致。另外, $\mathrm{BiOBr}$ 样品的(001)晶面衍射峰强度明显强于 $\mathrm{FBiOBr}$ ，表 明其具有高暴露的(001) 晶面 ${ }^{[21]}$ 。与其它晶面相比, (001) 晶面具有较高的表面能及较强的终端氧原子 密度, 能呈现出更好的电荷转移能力和催化活性 ${ }^{[22]}$ 。

BT- $x$ 和 FBT-20 复合催化剂都显示出 BiOBr 的特征 峰, 但未出现 $\mathrm{Ti}_{3} \mathrm{C}_{2}$ 的特征峰, 这是因为与 $\mathrm{BiOBr}$ 相 比, $\mathrm{Ti}_{3} \mathrm{C}_{2}$ 结晶性较差, 特征峰衍射强度较低。

\subsubsection{SEM 和 EDS 分析}

采用 SEM 观察 $\mathrm{BiOBr} 、 \mathrm{Ti}_{3} \mathrm{C}_{2}$ 和 $\mathrm{BT}-20$ 样品的
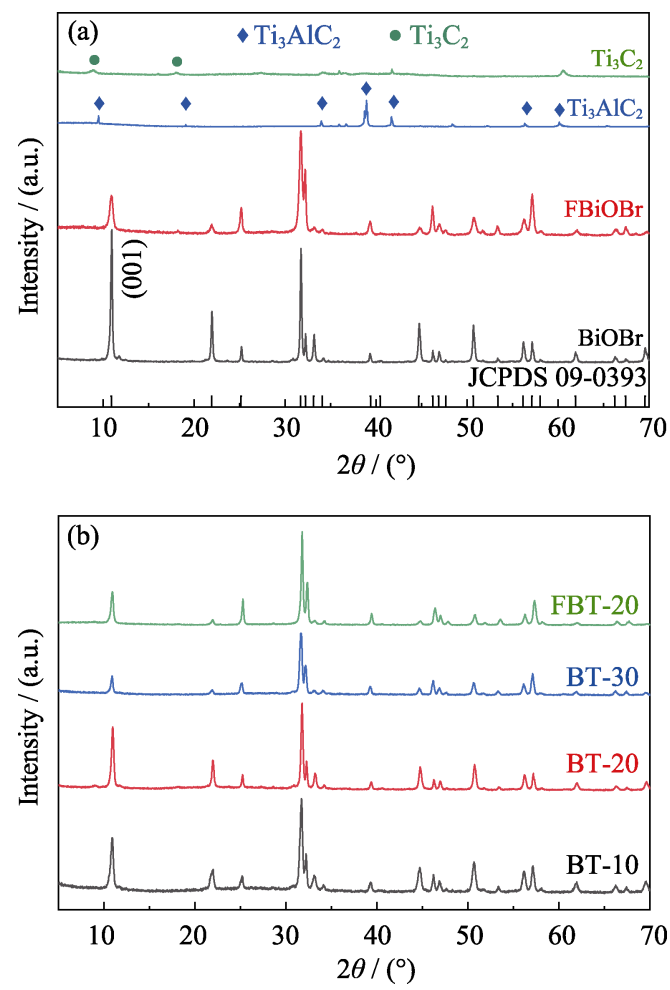

图 $1 \mathrm{Ti}_{3} \mathrm{C}_{2}, \mathrm{Ti}_{3} \mathrm{AlC}_{2}, \mathrm{FBiOBr}, \mathrm{BiOBr}(\mathrm{a})$ 和 FBT-20, BT- $x$ (b)样 品的 XRD 图谱

Fig. 1 XRD patterns of samples $\mathrm{Ti}_{3} \mathrm{C}_{2}, \mathrm{Ti}_{3} \mathrm{AlC}_{2}, \mathrm{FBiOBr}$, $\mathrm{BiOBr}(\mathrm{a})$ and samples FBT-20, BT- $x$ (b)

形貌。从图 2(a,b)中可以看出, $\mathrm{BiOBr}$ 样品呈二维薄 片状，堆叠生长，尺寸约为 $0.3 \sim 2.0 \mu \mathrm{m} ; \mathrm{Ti}_{3} \mathrm{C}_{2}$ 则呈 典型的手风琴结构。图 $2(\mathrm{c}, \mathrm{d})$ 直观地呈现了 $\mathrm{BiOBr}$ 与 $\mathrm{Ti}_{3} \mathrm{C}_{2}$ 的复合情况, 可以看到 $\mathrm{BiOBr}$ 堆叠生长于 $\mathrm{Ti}_{3} \mathrm{C}_{2}$ 表面两侧, 与 $\mathrm{Ti}_{3} \mathrm{C}_{2}$ 表面紧密结合。 $\mathrm{Ti}_{3} \mathrm{C}_{2}$ 为良 好的导电体 ${ }^{[12]}$, 可以为电子传递提供良好的通道, 有利于 $\mathrm{BiOBr}$ 的光生载流子的转移, 促进光生电 子-空穴对的分离 ${ }^{[15]}$ 。由图 2(e)所示, 通过 EDS 对 BT-20 样品进行元素扫描, 发现 $\mathrm{C} 、 \mathrm{O} 、 \mathrm{Ti} 、 \mathrm{Br}$ 和 $\mathrm{Bi}$ 元素均匀分布, 进一步表明实验成功制备了 $\mathrm{BiOBr} / \mathrm{Ti}_{3} \mathrm{C}_{2}$ 复合光催化剂。

\subsubsection{TEM/HRTEM 分析}

采用 TEM/HRTEM 进一步研究 BiOBr 的微观结 构。图 3(a)为 $\mathrm{BiOBr}$ 的 TEM 照片, 可以看出 $\mathrm{BiOBr}$ 的厚度较薄, 呈现薄片状。由图 3(b)的 HRTEM 照 片可以清晰地看到，两组晶面间距为 $0.277 \mathrm{~nm}$ 的晶 格条纹相互垂直, 分别对应 $\mathrm{BiOBr}$ 的(110)和 (1110) 面。由图 3(c)的选区电子衍射(SAED) 可知, (110)和 (200)面成 $45^{\circ}$ 夹角, 该角度值与 $\mathrm{BiOBr}$ 的晶面角理 论值相符。因此 $\mathrm{BiOBr}$ 片层表面垂直方向为 [001] 晶轴方向, 纳米片的上下表面为(001)晶面, 侧面为 (110)和 (11 10$)$ 晶面(图 3(d) $)^{[23]}$ 。晶体在生长过程中总 是趋于降低晶体的总表面能, 因此纳米片会尽量地 

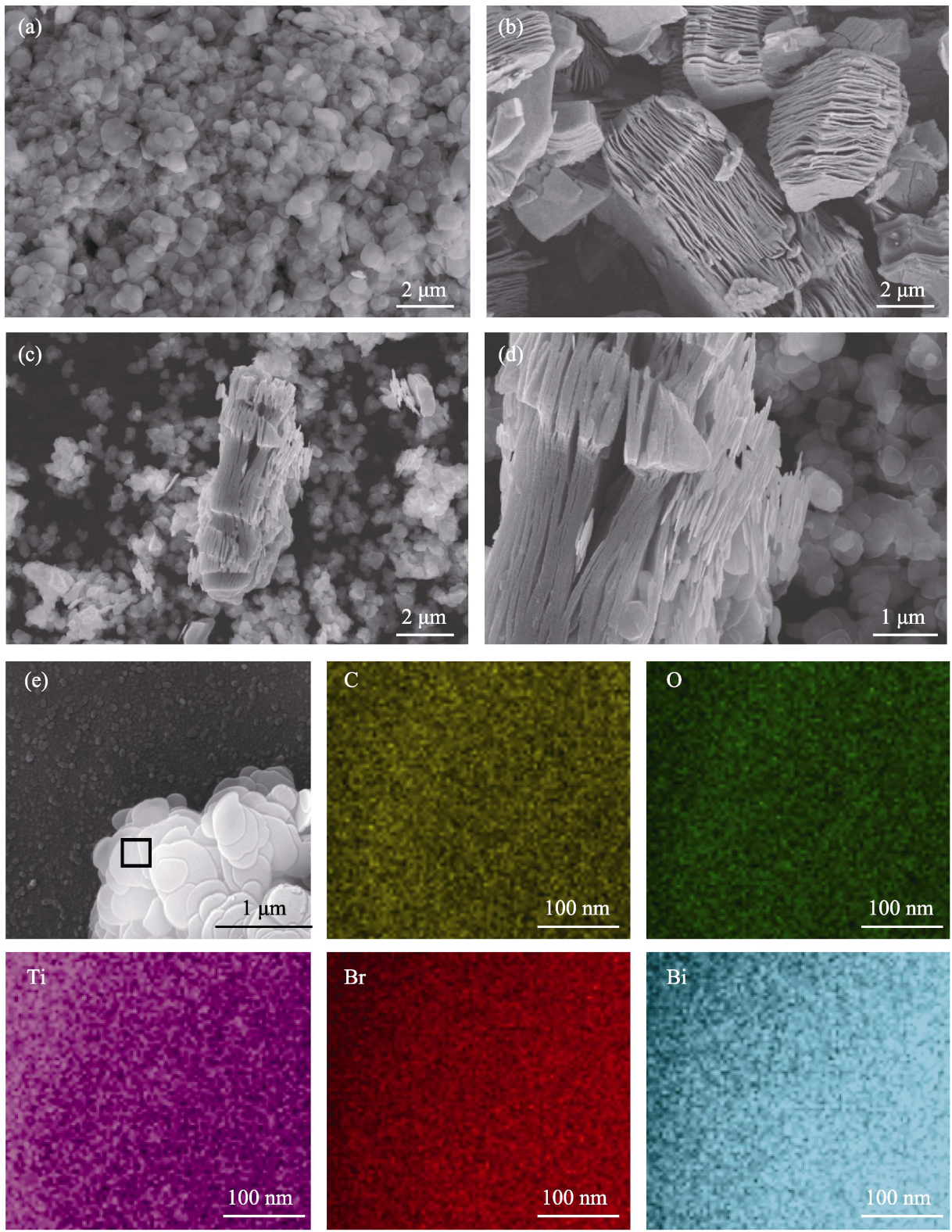

图 $2 \operatorname{BiOBr}(\mathrm{a}), \mathrm{Ti}_{3} \mathrm{C}_{2}(\mathrm{~b}), \mathrm{BT}-20(\mathrm{c} \sim \mathrm{d})$ 的 SEM 照片和 BT-20(e)的 SEM 照片及其元素的 EDS 面分布

Fig. 2 SEM images of $\mathrm{BiOBr}(\mathrm{a}), \mathrm{Ti}_{3} \mathrm{C}_{2}$ (b), BT-20 (c-d) and SEM-EDS elemental mapping of BT-20 (e)
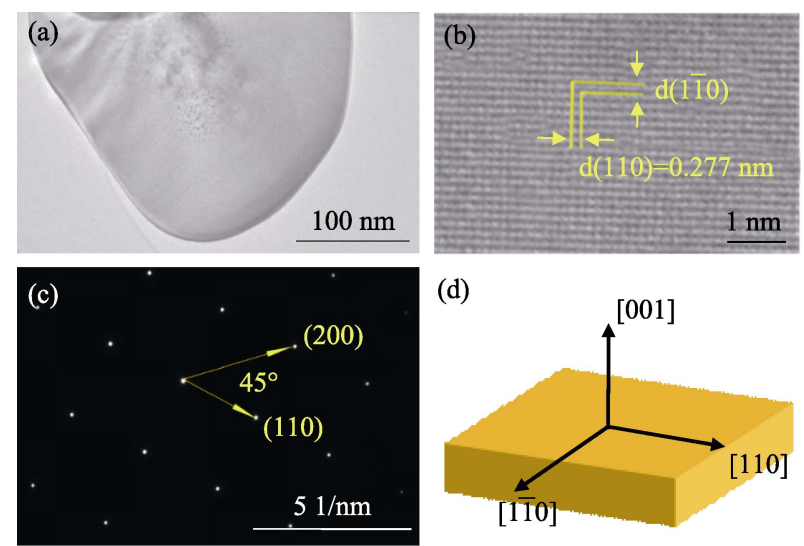

(d)

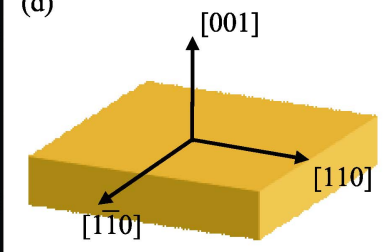

图 $3 \mathrm{BiOBr}$ 样品的 TEM 照片(a), HRTEM 照片(b), SAED 图 案(c)以及晶体生长方向机理图(d)

Fig. 3 TEM/HRTEM images (a-b), SAED pattern (c) and schematic illustrations of crystal orientation (d) of BiOBr sample
隐藏高表面能的晶面来获得总表面能较低的晶体结 构 ${ }^{[24]}$ 。对于具有高暴露(001) 晶面的 $\mathrm{BiOBr}$ 纳米片样 品来说, (001) 高能晶面倾向于堆叠来减少它的暴露, 这与 SEM 的分析结果一致。

\subsubsection{XPS 分析}

图 4 为 $\mathrm{BiOBr}$ 和 $\mathrm{BT}-20$ 样品的 XPS 图谱, 由 BT-20 的 XPS 全谱图可以清晰地观察到存在 Br、O、 $\mathrm{Bi}$ 和 $\mathrm{Ti}$ 元素, 这与 $\mathrm{EDS}$ 结果一致。与 $\mathrm{BiOBr}$ 相比, BT-20 的 Br2d、Bi4f、O1s 的出峰位置所对应的结 合能有所增大，这可能是由于引入高导电性 $\mathrm{Ti}_{3} \mathrm{C}_{2}$ 导致 $\mathrm{BiOBr}$ 周围的电荷密度降低, 从而增强了复合 催化剂表面的结合能 ${ }^{[13]}$ 。当 $\mathrm{Ti}_{3} \mathrm{C}_{2}$ 与 $\mathrm{BiOBr}$ 接触后, 光生电子的转移路径从 $\mathrm{BiOBr}$ 导带处转移至 $\mathrm{Ti}_{3} \mathrm{C}_{2}$ 表面, 促进界面肖特基结能垒的形成, 进一步在 

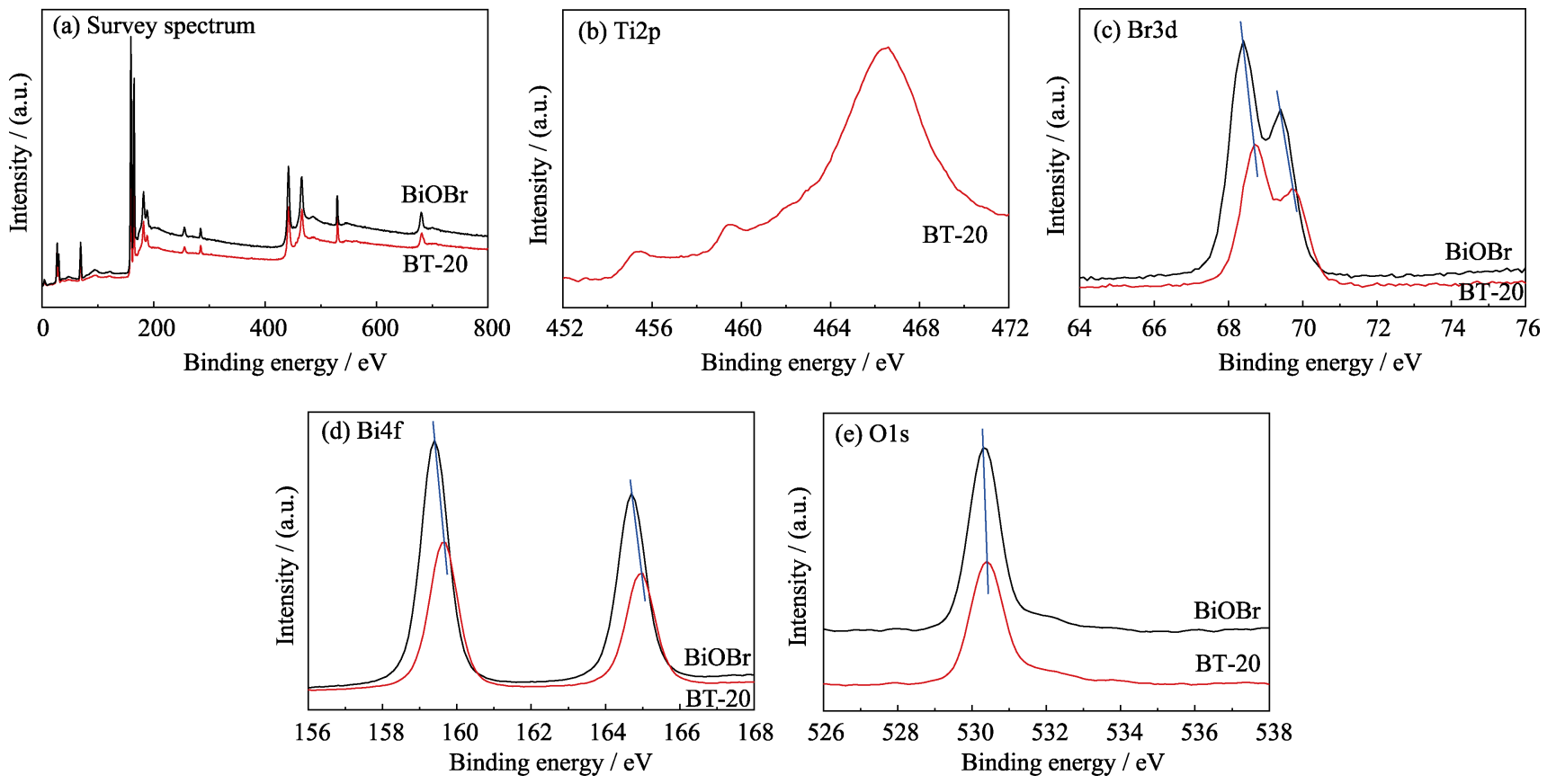

图 $4 \mathrm{BiOBr}$ 和 BT-20 样品的 XPS 谱图

Fig. 4 XPS spectra of samples BiOBr and BT-20

(a) Survey spectra; (b) Ti2p; (c) Br3d; (d) Bi4f; (f) O1s

$\mathrm{BiOBr}$ 与 $\mathrm{Ti}_{3} \mathrm{C}_{2}$ 的界面间形成内建电场，定向转移光 生电子，有效地抑制光生载流子的复合，从而提高 了光催化剂的光催化活性 ${ }^{[25]}$ 。

\subsection{5 光学性质分析}

通过紫外可见漫反射图谱研究催化剂的光学性 质。由图 5(a)可知, 样品具有吸收紫外和可见光的 能力, $\mathrm{BiOBr}$ 的吸收边位于 $430 \mathrm{~nm}$ 处, 引入层状 $\mathrm{Ti}_{3} \mathrm{C}_{2}$ 后, $\mathrm{BiOBr} / \mathrm{Ti}_{3} \mathrm{C}_{2}$ 复合光催化剂的吸收边红移至 $460 \mathrm{~nm}$, 且在可见光区具有更强的吸收, 这表明引 入层状 $\mathrm{Ti}_{3} \mathrm{C}_{2}$ 拓宽了 $\mathrm{BiOBr}$ 的可见光响应区域。禁 带宽度 $E_{\mathrm{g}}$ 可由下式进行计算 ${ }^{[26]}$.

$$
\alpha h v=A\left(h v-E_{\mathrm{g}}\right)^{n / 2}
$$

其中, $n$ 值由半导体催化剂的带间跃迁类型决定, 直 接跃迁型 $n$ 为 1 , 间接跃迁型 $n$ 为 4 。由于 $\mathrm{BiOBr}$ 的跃迁属于间接跃迁型, 即 $n$ 为 $4^{[27]}$ 。由图 5(b) 可知, $\mathrm{BiOBr}$ 和 BT-20 的禁带宽度分别为 2.75 和 $2.40 \mathrm{eV}$, BT-20 禁带宽度小于 $\mathrm{BiOBr}$, 表明 $\mathrm{BiOBr} / \mathrm{Ti}_{3} \mathrm{C}_{2}$ 复合 光催化剂具有更好的可见光响应能力。

通过光致发光光谱研究光催化剂光生电子-空 穴对的转移和重组行为。由图 6 可知, 与 $\mathrm{BiOBr}$ 相 比, $\mathrm{BiOBr} / \mathrm{Ti}_{3} \mathrm{C}_{2}$ 复合光催化剂的苂光强度明显降低, 其中, BT-20 的苂光强度最低, 这表明引入适量具有 高导电性的 $\mathrm{Ti}_{3} \mathrm{C}_{2}$ 能转移光生电子, 促进光生电子空穴对的分离。

\section{2 可见光催化降解性能}

通过在可见光 $(\lambda>420 \mathrm{~nm})$ 照射下降解 $\mathrm{RhB}$ 溶液
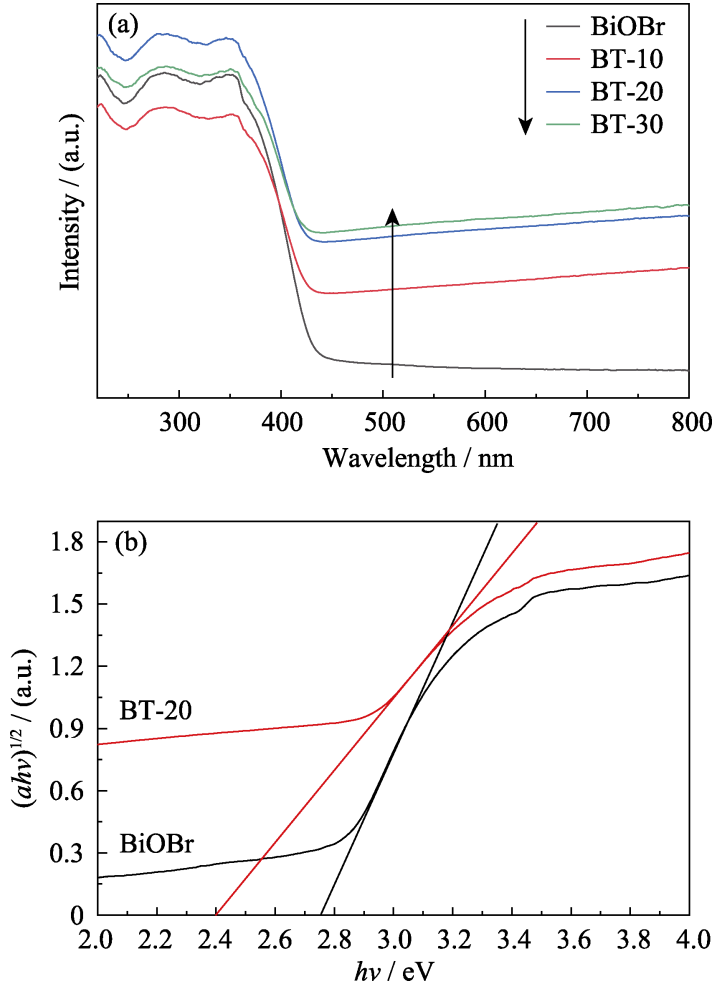

图 $5 \mathrm{BiOBr}$ 和 BT- $x$ 样品的紫外可见漫反射光谱(a)及 $(\alpha h v)^{1 / 2}-h v$ 曲线(b)

Fig. 5 UV-Vis diffuse reflectance spectra (a) and $(\alpha h v)^{1 / 2}-h v$ curves (b) of samples BiOBr and BT- $x$

来评价 $\mathrm{BiOBr} / \mathrm{Ti}_{3} \mathrm{C}_{2}$ 复合光催化剂的光催化活性。 如图 7(a)所示, 在无催化剂的对照实验中, RhB 几乎 没有发生降解。BT-20 复合材料的光催化降解性能 


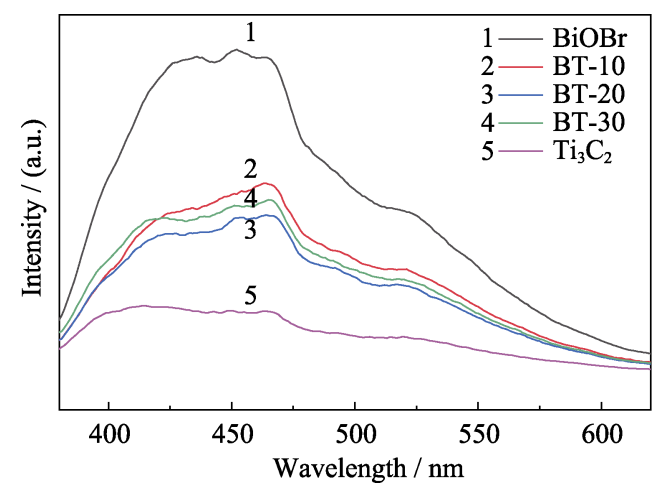

图 $6 \mathrm{BiOBr}$ 和 BT- $x$ 样品的 PL 图谱

Fig. 6 PL spectra of samples BiOBr and BT- $x$
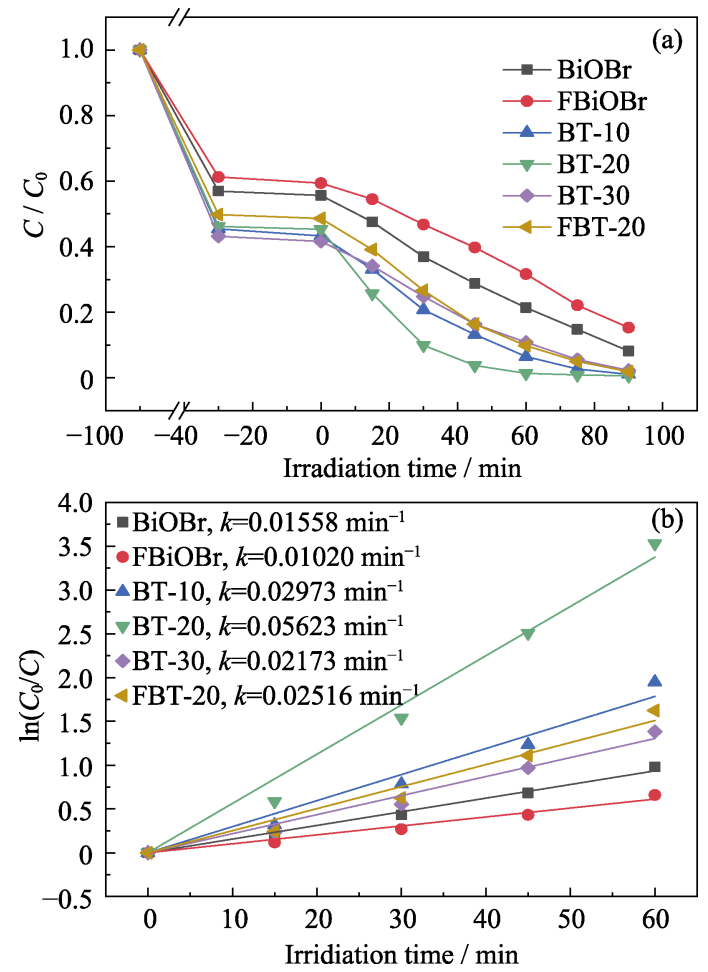

图 $7 \mathrm{BiOBr}$ 和 BT- $x$ 样品的可见光催化降解 $\mathrm{RhB}(\mathrm{a})$ 及伪一 级动力学曲线(b)

Fig. 7 Photocatalytic degradation activity of samples $\mathrm{BiOBr}$ and BT- $x$ for RhB (a) and pseudo first-order kinetic curves (b) under visible light irradiation

最高, 光照 $60 \mathrm{~min}$ 后其对 $\mathrm{RhB}$ 的降解率可达 $97.1 \%$, 比 $\mathrm{BiOBr} 、 \mathrm{FBiOBr} 、 \mathrm{FBT}-20$ 分别提高了 $34.7 \%$ 、 $48.8 \% 、 16.81 \%$ 。随着 $\mathrm{Ti}_{3} \mathrm{C}_{2}$ 引入量的增加, $\mathrm{BT}$ 系列 样品中 $\mathrm{BiOBr}$ 的捕光能力有所下降, 由此可见, 引 入适量 $\mathrm{Ti}_{3} \mathrm{C}_{2}$ 可使 $\mathrm{BiOBr} / \mathrm{Ti}_{3} \mathrm{C}_{2}$ 达到最佳的电荷分离 效率和捕光能力 ${ }^{[16]}$ 。

为了定量研究样品的光催化活性, 采用伪一级 动力学方程 $\ln \left(C / C_{0}\right)=k t$ 对样品光催化降解 $\mathrm{RhB}$ 的 反应进行拟合, 其中 $k$ 为降解速率常数 $\left(\mathrm{min}^{-1}\right)^{[28]}$ 。 图 7(b)中 BiOBr、FBiOBr、BT-10、BT-20、BT-30、 FBT-20 六条拟合曲线相关系数分别为 0.9952 、
$0.9901 、 0.9862 、 0.9934 、 0.9905 、 0.9858$, 符合伪一 级动力学模型 ${ }^{[29]}$. 如图 7(b) 所示, $\mathrm{BiOBr}$ 的降解速率 常数为 $0.01558 \mathrm{~min}^{-1}$, 是 $\mathrm{FBiOBr}$ 的 1.5 倍, 表明 $\mathrm{BiOBr}$ 光催化活性优于 $\mathrm{FBiOBr}$, 这是由于高暴露 (001)面可以呈现出更好的光生电子-空穴对的迁移

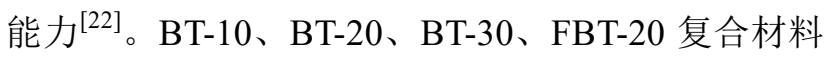
光催化降解速率常数分别为 $0.02973 、 0.05623$ 、 $0.02173 、 0.02516 \mathrm{~min}^{-1}$, 均高于 $\mathrm{BiOBr}$, 其中 BT-20 的降解速率常数分别是 $\mathrm{BiOBr}$ 和 FBT-20 的 3.6 和 2.2 倍。结果表明, 引入 $\mathrm{Ti}_{3} \mathrm{C}_{2}$ 使得 $\mathrm{BiOBr}$ 与 $\mathrm{Ti}_{3} \mathrm{C}_{2}$ 界面形成肖特基结势垒, 促进 $\mathrm{BiOBr} / \mathrm{Ti}_{3} \mathrm{C}_{2}$ 复合材 料光生电子-空穴对的分离, 从而大大提高了其可 见光催化性能。

\section{3 稳定性测试}

光催化剂的稳定性也是评价其性能的一项重要 指标。为考察复合光催化剂的稳定性, 对催化剂进 行了再循环实验。循环实验与降解实验步骤一致, 光降解 $90 \mathrm{~min}$ 后, 通过离心回收催化剂, 进行下一 次实验。如图 8 所示, 经过 5 次循环后, 催化剂对 $\mathrm{RhB}$ 降解率仍高达 $91.0 \%$, 表明 BT-20 复合光催化 剂具有良好的稳定性。

\section{4 机理分析}

为了探究 $\mathrm{BiOBr} / \mathrm{Ti}_{3} \mathrm{C}_{2}$ 复合材料的光催化机理, 通过自由基和空穴捕获实验确定其在可见光下降解 $\mathrm{RhB}$ 过程中的主要活性物种 ${ }^{[30]}$ 。选用一水合草酸铵 $(\mathrm{AO})$ 作为空穴 $\left(\mathrm{h}^{+}\right)$捕获剂 ${ }^{[31]}$, 苯醌 (BQ) 作为超氧自 由基 $\left(\cdot \mathrm{O}_{2}{ }^{-}\right)$捕获剂 ${ }^{[32]}$, 异丙醇 $(\mathrm{IPA})$ 作为羟基自由基 $(\cdot \mathrm{OH})$ 捕获剂 ${ }^{[33]}$, 捕获剂添加量均为 $5 \mathrm{mmol} / \mathrm{L}$ 。三种 捕获剂对催化剂降解性能的影响如图 9 所示。当加 入 IPA 后, BT-20 复合光催化剂的光催化活性几乎没 有受到影响; 加入 BQ 后, BT-20 的降解性能受到显 著影响, 反应 $90 \mathrm{~min}$ 后 $\mathrm{RhB}$ 降解率减少了 $68.0 \%$; 加 入 $\mathrm{AO}$ 后, $\mathrm{RhB}$ 的降解率减少 $26.0 \%$ 。由此可见, 在

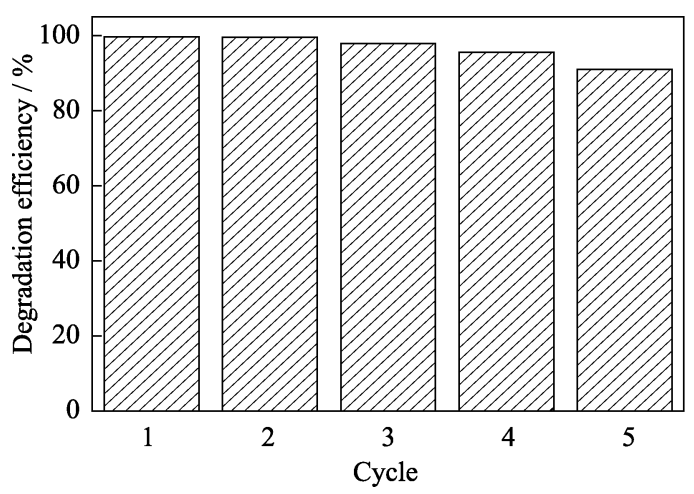

图 8 在可见光照射下 BT-20 光催化降解 RhB 的循环性能 Fig. 8 Cycle performance of BT-20 for photodegradation of $\mathrm{RhB}$ under visible light irradiation 


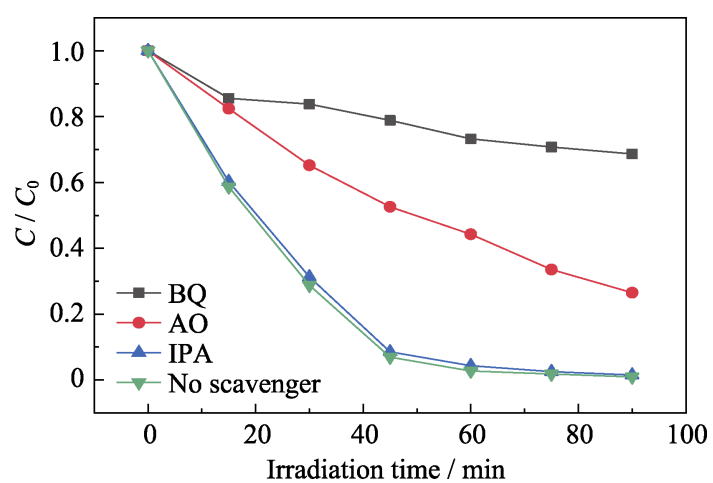

图 9 可见光照射下 BT-20 光催化降解 RhB 的活性物种捕获 结果

Fig. 9 Effect of scavengers on the degradation of RhB with BT-20 under visible light irradiation

BT-20 复合材料光催化降解 $\mathrm{RhB}$ 的过程中, $\cdot \mathrm{O}_{2}{ }^{-}$为其 主要活性物种, $\mathrm{h}^{+}$为次要活性物种, $\cdot \mathrm{OH}$ 作用最小。

通过经验公式计算 $\mathrm{BiOBr}$ 的价带电势 $\left(E_{\mathrm{VB}}\right)$ 和导 带电势 $\left(E_{\mathrm{CB}}\right)^{[34]}$ :

$$
\begin{gathered}
E_{\mathrm{VB}}=X-E_{\mathrm{e}}+0.5 E_{\mathrm{g}} \\
E_{\mathrm{CB}}=E_{\mathrm{VB}}-E_{\mathrm{g}}
\end{gathered}
$$

其中, $X$ 为绝对电负性, $\mathrm{BiOBr}$ 的绝对电负性为 $6.18 \mathrm{eV}^{[35]} ; E_{\mathrm{e}}$ 为自由电子在标准氢电极的势能, 约 为 $4.50 \mathrm{eV} ; E_{\mathrm{g}}$ 为半导体催化剂的禁带宽度, $\mathrm{BiOBr}$ 的 $E_{\mathrm{g}}$ 为 $2.75 \mathrm{eV}$ 。由计算可得, $\mathrm{BiOBr}$ 的 $E_{\mathrm{VB}}$ 和 $E_{\mathrm{CB}}$ 分别 为 3.06 和 $0.31 \mathrm{eV}$ 。

$\mathrm{BiOBr}$ 属于 $\mathrm{n}$ 型半导体, $\mathrm{BiOBr}$ 的费米能级靠近 导带, 当两者形成紧密的界面接触并在可见光的照 射下, $\mathrm{Ti}_{3} \mathrm{C}_{2}$ 和 $\mathrm{BiOBr}$ 之间的费米能级差驱使 $\mathrm{BiOBr}$ 导带处的电子迁移至 $\mathrm{Ti}_{3} \mathrm{C}_{2}$ 处, 直至两者费米能级 达到平衡, 并伴随着导带和价带能级向上弯曲, 在 界面处形成界面肖特基结能垒 ${ }^{[36-37]}$ 。

基于以上分析, $\mathrm{BiOBr} / \mathrm{Ti}_{3} \mathrm{C}_{2}$ 肖特基结光催化剂 的光催化机理可由图 10 所示。在可见光照射下, $\mathrm{BiOBr}$ 激发产生光生电子-空穴对, 形成内建电场促 使 $\mathrm{BiOBr}$ 导带处的光生电子单向跨越肖特基结能垒

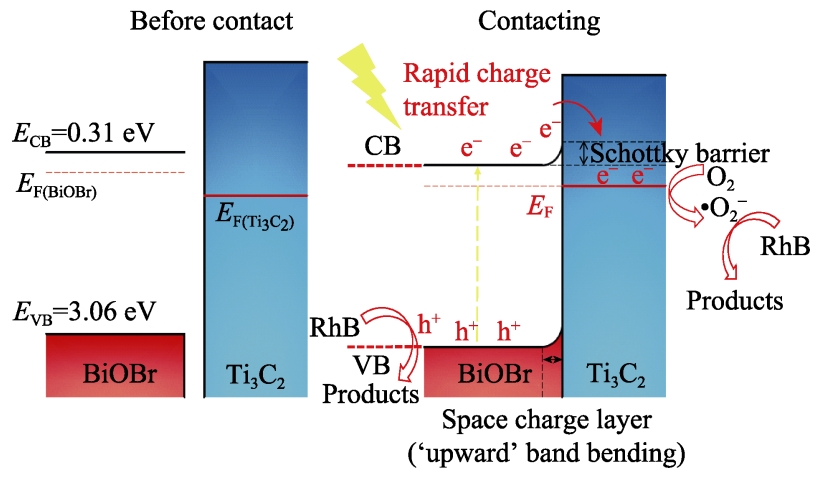

图 $10 \mathrm{BiOBr} / \mathrm{Ti}_{3} \mathrm{C}_{2}$ 体系可见光催化降解 $\mathrm{RhB}$ 的机理

Fig.10 Photocatalytic mechanism of the $\mathrm{BiOBr} / \mathrm{Ti}_{3} \mathrm{C}_{2}$ composite on $\mathrm{RhB}$
流入 $\mathrm{Ti}_{3} \mathrm{C}_{2}$ 捕获场, 从而有效地分离载流子, 迁移至 $\mathrm{Ti}_{3} \mathrm{C}_{2}$ 处的光生电子将 $\mathrm{O}_{2}$ 还原为 $\cdot \mathrm{O}_{2}^{-}, \cdot \mathrm{O}_{2}$ 继而氧 化 $\mathrm{RhB}$, 同时 $\mathrm{BiOBr}$ 价带处具有强氧化性的空穴直 接氧化降解 $\mathrm{RhB}$ 。

\section{3 结论}

通过水解法对具有高暴露(001)面的 $\mathrm{BiOBr}$ 与 $\mathrm{Ti}_{3} \mathrm{C}_{2}$ 进行复合, 制备出一系列高暴露 (001) 面 $\mathrm{BiOBr} / \mathrm{Ti}_{3} \mathrm{C}_{2}$ 复合光催化剂。与 $\mathrm{BiOBr}$ 相比, $\mathrm{BT}-20$ 复合光催化剂降解 RhB 的效果最优, 降解速率常数 为 $\mathrm{BiOBr}$ 的 3.6 倍, 并具有良好的循环稳定性。机 理分析表明 $\mathrm{BiOBr}$ 与 $\mathrm{Ti}_{3} \mathrm{C}_{2}$ 界面形成肖特基结能垒, 促进光生电子快速向 $\mathrm{Ti}_{3} \mathrm{C}_{2}$ 转移, 抑制了光生电子空穴对的复合，从而提高了 $\mathrm{BiOBr}$ 的光催化活性。 本研究合成的高暴露 $(001)$ 面 $\mathrm{BiOBr} / \mathrm{Ti}_{3} \mathrm{C}_{2}$ 光催化剂, 为构建新型高效的肖特基结光催化剂体系提供了一 种可行的途径。

\section{参考文献:}

[1] LU H, CHEN A P, SUN X L, et al. Synthesis of graphene/ $\mathrm{Ni} / \mathrm{TiO}_{2} / \mathrm{CNTs}$ composites and photocatalytic activities. Chinese Journal of Inorganic Chemistry, 2014, 29(10): 1062-1066.

[2] ETACHERI V, DI VALENTIN C, SCHNEIDER J, et al. Visiblelight activation of $\mathrm{TiO}_{2}$ photocatalysts: advances in theory and experiments. Journal of Photochemistry and Photobiology C: Photochemistry Reviews, 2015, 25: 1-29.

[3] CHEN X B, LIU L, HUANG F Q. Black titanium dioxide $\left(\mathrm{TiO}_{2}\right)$ nanomaterials. Chemical Society Reviews, 2015, 44(7): 1861-1885.

[4] LI J, YU Y, ZHANG L Z. Bismuth oxyhalide nanomaterials: layered structures meet photocatalysis. Nanoscale, 2014, 6(15): 8473-8488.

[5] ZHANG K L, LIU C M, HUANG F Q, et al. Study of the electronic structure and photocatalytic activity of the $\mathrm{BiOCl}$ photocatalyst. Applied Catalysis B: Environmental, 2006, 68: 125-129.

[6] HUANG W L, ZHU Q S. Electronic structures of relaxed BiOX ( $\mathrm{X}=\mathrm{F}, \mathrm{Cl}, \mathrm{Br}$, I) photocatalysts. Computational Materials Science, 2008, 43(4): 1101-1108.

[7] GUO W, QIN Q, GENG L, et al. Morphology-controlled preparation and plasmon-enhanced photocatalytic activity of $\mathrm{Pt}-\mathrm{BiOBr}$ heterostructures. Journal of Hazardous Materials, 2016, 308: 374-385.

[8] XIONG X, DING L, WANG Q, et al. Synthesis and photocatalytic activity of BiOBr nanosheets with tunable exposed $\{010\}$ facets. Applied Catalysis B: Environmental, 2016, 188: 283-291.

[9] ZHANG H, YANG Y, ZHOU Z, et al. Enhanced photocatalytic properties in $\mathrm{BiOBr}$ nanosheets with dominantly exposed $\{102\}$ facets. Journal of Physical Chemistry C, 2014, 118(26): 14662-14669.

[10] CHENG H F, HUANG B B, WANG P, et al. In situ ion exchange synthesis of the novel $\mathrm{Ag} / \mathrm{AgBr} / \mathrm{BiOBr}$ hybrid with highly efficient decontamination of pollutants. Chemical Communication, 2011, 47(25): 7054-7056.

[11] AI Z, HO W, LEE S. Efficient visible light photocatalytic removal of NO with BiOBr-graphene nanocomposites. Journal of Physical Chemistry C, 2011, 115(51): 25330-25337. 
[12] KAJIYAMA S, SZABOVA L, SODEYAMA K, et al. Sodium-ion intercalation mechanism in MXene nanosheets. ACS Nano, 2016, 10(3): 3334-3341.

[13] ZHUANG Z, LI Y, LI F, et al. $\mathrm{MoB} / \mathrm{g}-\mathrm{C}_{3} \mathrm{~N}_{4}$ interface materials as a schottky catalyst to boost hydrogen evolution. Angewandte Chemie International Edition, 2018, 57(2): 496-500.

[14] PENG C, YANG X F, LI Y H, et al. Hybrids of two-dimensional $\mathrm{Ti}_{3} \mathrm{C}_{2}$ and $\mathrm{TiO}_{2}$ exposing $\{001\}$ facets toward enhanced photocatalytic activity. ACS Applied Materials \& Interfaces, 2016, 8(9): 6051-6060.

[15] CAI T, WANG L L, LIU Y T, et al. $\mathrm{Ag}_{3} \mathrm{PO}_{4} / \mathrm{Ti}_{3} \mathrm{C}_{2}$ MXene interface materials as a schottky catalyst with enhanced photocatalytic activities and anti-photocorrosion performance. Applied Catalysis B: Environmental, 2018, 239: 545-554.

[16] LIU C, XU Q X, ZHANG Q F, et al. Layered BiOBr/Ti ${ }_{3} \mathrm{C}_{2}$ MXene composite with improved visible-light photocatalytic activity. Journal of Materials Science, 2019, 54(3): 2458-2471.

[17] HUANG Q S, LIU Y T, CAI T, et al. Simultaneous removal of heavy metal ions and organic pollutant by $\mathrm{BiOBr} / \mathrm{Ti}_{3} \mathrm{C}_{2}$ nanocomposite. Journal of Photochemistry and Photobiology A-Chemistry, 2019, 375: 201-208.

[18] LI Z Z, ZHANG H G, WANG L, et al. 2D/2D BiOBr/ $/ \mathrm{Ti}_{3} \mathrm{C}_{2}$ heterojunction with dual applications in both water detoxification and water splitting. Journal of Photochemistry and Photobiology A-Chemistry, 2020, 386: 112099.

[19] LI Y J, DENG X T, TIAN J, et al. $\mathrm{Ti}_{3} \mathrm{C}_{2}$ MXene-derived $\mathrm{Ti}_{3} \mathrm{C}_{2} / \mathrm{TiO}_{2}$ nanoflowers for noble-metal-free photocatalytic overall water splitting. Applied Materials Today, 2018, 13: 217-227.

[20] YANG C, LIU Y, SUN X, et al. In-situ construction of hierarchical accordion-like $\mathrm{TiO}_{2} / \mathrm{Ti}_{3} \mathrm{C}_{2}$ nanohybrid as anode material for lithium and sodium ion batteries. Electrochimica Acta, 2018, 271: 165-172.

[21] YE L Q, TIAN L H, PENG T Y, et al. Synthesis of highly symmetrical BiOI single-crystal nanosheets and their $\{001\}$ facetdependent photoactivity. Journal of Materials Chemistry, 2011, 21(33): 12479-12484.

[22] LI J, YU Y, ZHANG L Z. Bismuth oxyhalide nanomaterials: layered structures meet photocatalysis. Nanoscale, 2014, 6(15): $8473-8488$.

[23] LU L, ZHOU M Y, YIN L, et al. Tuning the physicochemical property of $\mathrm{BiOBr}$ via $\mathrm{pH}$ adjustment: towards an efficient photocatalyst for degradation of bisphenol A. Journal of Molecular Catalysis A-Chemical, 2016, 423: 379-385.

[24] ZHU L P, LIAO G H, BING N C, et al. Self-assembled 3D BiOCl hierarchitectures: tunable synthesis and characterization. Crystengcomm, 2010, 12(11): 3791-3796.

[25] BAI S, JIANG J, ZHANG Y, et al. Steering charge kinetics in photocatalysis: intersection of materials syntheses, characterization techniques and theoretical simulations. Chemical Society Reviews,
2015, 44(10): 2893-2939.

[26] LI K, HUANG Z, ZENG X, et al. Synergetic effect of $\mathrm{Ti}^{3+}$ and oxygen doping on enhancing photoelectrochemical and photocatalytic properties of $\mathrm{TiO}_{2} / \mathrm{g}-\mathrm{C}_{3} \mathrm{~N}_{4}$ heterojunctions. ACS Applied Materials \& Interfaces, 2017, 9(13): 11577-11586.

[27] ZHANG K L, LIU C M, HUANG F Q, et al. Study of the electronic structure and photocatalytic activity of the $\mathrm{BiOCl}$ photocatalyst. Applied Catalysis B: Environmental, 2006, 68: 125-129.

[28] LI W, LI C, CHEN B, et al. Facile synthesis of sheet-like $\mathrm{N}-\mathrm{TiO}_{2} / \mathrm{g}-\mathrm{C}_{3} \mathrm{~N}_{4}$ heterojunctions with highly enhanced and stable visible-light photocatalytic activities. RSC Advances, 2015, 5(43): 34281-34291.

[29] GE L, HAN C C, LIU J. Novel visible light-induced g- $\mathrm{C}_{3} \mathrm{~N}_{4} / \mathrm{Bi}_{2} \mathrm{WO}_{6}$ composite photocatalysts for efficient degradation of methyl orange. Applied Catalysis B: Environmental, 2011, 108: $100-107$.

[30] WANG F Z, LI W J, GU S N, et al. Visible-light-driven heterojunction photocatalysts based on $\mathrm{g}-\mathrm{C}_{3} \mathrm{~N}_{4}$ decorated $\mathrm{La}_{2} \mathrm{Ti}_{2} \mathrm{O}_{7}$ : effective transportation of photogenerated carriers in this heterostructure. Catalysis Communications, 2017, 96: 50-53.

[31] WANG J, TANG L, ZENG G, et al. Atomic scale g- $\mathrm{C}_{3} \mathrm{~N}_{4} / \mathrm{Bi}_{2} \mathrm{WO}_{6}$ $2 \mathrm{D} / 2 \mathrm{D}$ heterojunction with enhanced photocatalytic degradation of ibuprofen under visible light irradiation. Applied Catalysis B: Environmental, 2017, 209: 285-294.

[32] LIU C, ZHU H, ZHU Y, et al. Ordered layered N-doped $\mathrm{KTiNbO}_{5} / \mathrm{g}-\mathrm{C}_{3} \mathrm{~N}_{4}$ heterojunction with enhanced visible light photocatalytic activity. Applied Catalysis B: Environmental, 2018, 228: $54-63$

[33] WAN Z, ZHANG G, WU X, et al. Novel visible-light-driven $\mathrm{Z}$-scheme $\mathrm{Bi}_{12} \mathrm{GeO}_{20} / \mathrm{g}-\mathrm{C}_{3} \mathrm{~N}_{4}$ photocatalyst: oxygen-induced pathway of organic pollutants degradation and proton assisted electron transfer mechanism of $\mathrm{Cr}(\mathrm{VI})$ reduction. Applied Catalysis B: Environmental, 2017, 207: 17-26.

[34] YE L Q, LIU J Y, GONG C Q, et al. Two different roles of metallic $\mathrm{Ag}$ on $\mathrm{Ag} / \mathrm{AgX} / \mathrm{BiOX}(\mathrm{X}=\mathrm{Cl}, \mathrm{Br})$ visible light photocatalysts: surface plasmon resonance and Z-Scheme bridge. ACS Catalysis, 2012, 2(8): 1677-1683

[35] CHEN S F, HU Y F, MENG S, et al. Study on the separation mechanisms of photogenerated electrons and holes for composite photocatalysts g- $\mathrm{C}_{3} \mathrm{~N}_{4}-\mathrm{WO}_{3}$. Applied Catalysis B: Environmental, 2014, 150: 564-573.

[36] ZHANG Z, YATES J T. Band bending in semiconductors: chemical and physical consequences at surfaces and interfaces. Chemical Reviews, 2012, 112(10): 5520-5551.

[37] YANG Y, ZENG Z T, ZENG G M, et al. $\mathrm{Ti}_{3} \mathrm{C}_{2}$ Mxene/porous $\mathrm{g}_{-} \mathrm{C}_{3} \mathrm{~N}_{4}$ interfacial schottky junction for boosting spatial charge separation in photocatalytic $\mathrm{H}_{2} \mathrm{O}_{2}$ production. Applied Catalysis B: Environmental, 2019, 258: 117956. 